\title{
Increased activated regulatory T cells proportion correlate with the severity of idiopathic pulmonary fibrosis
}

\author{
Ziliang Hou', Qiao Ye ${ }^{1 *}$ D, Meihua Qiu', Yu Hao ${ }^{2,3}$, Junyan Han ${ }^{2,3}$ and Hui Zeng ${ }^{2,3^{*}}$
}

\begin{abstract}
Background: Regulatory T cells (Tregs) are crucial in maintaining immune tolerance and immune homeostasis, but their role in idiopathic pulmonary fibrosis (IPF) is unclear. This study was designed to explore the role of Tregs in IPF.

Methods: Percentages of Tregs and their subpopulations in peripheral blood (PB) and bronchoalveolar lavage (BAL) samples were determined by flow cytometry in 29 patients with IPF, 19 patients with primary Sjögren's syndrome-related interstitial pneumonia (pSS-IP), and 23 healthy controls (HCs).

Results: In peripheral blood, no difference was found in $\mathrm{CD}^{+} \mathrm{CD} 25^{+} \mathrm{Foxp} 3^{+}$Treg percentages among patients with IPF, pSS-IP, or HCs. However, activated Treg (aTreg) fractions among CD4 ${ }^{+} \mathrm{T}$ cells increased significantly in IPF compared with pSS-IP or HCs. Being consistent with the result from the PB, aTreg fractions among CD4 ${ }^{+} T$ cells in IPF also increased significantly compared with pSS-IP or HCS, accompanied by increased fraction III compared with HCs in BAL. IPF patients had lower levels of resting Tregs (rTregs) from the thymus than did HCs, whereas aTreg levels originating from the thymus did not significantly differ from HCs. Both rTregs and aTregs proliferated in IPF, with aTregs being more proliferative than rTregs. Both rTregs and aTregs significantly inhibited proliferation of $\mathrm{CD}^{+} \mathrm{T}$ lymphocytes in vitro. The percentage of aTregs was correlated negatively with predicted diffusing capacity values for carbon monoxide and positively with GAP index in IPF.
\end{abstract}

Conclusions: Our study showed the imbalance between subpopulations of Tregs in IPF. Increased aTregs proportion in the peripheral blood correlated inversely with disease severity.

Keywords: Idiopathic pulmonary fibrosis, Interstitial pneumonia, Primary Sjögren's syndrome, Regulatory T cells

\section{Background}

Idiopathic pulmonary fibrosis (IPF) is a progressive and fatal chronic lung fibrosis disease of unknown cause with the histopathological features of usual interstitial pneumonia (UIP) [1]. Accumulated evidence indicates that IPF results from abnormal behaviour of the alveolar epithelial cells that provoke migration, proliferation, and activation of mesenchymal cells, causing the formation of fibroblastic and myofibroblastic foci as well as the destruction of the lung architecture [2].

\footnotetext{
* Correspondence: yeqiao_chaoyang@sina.com; zenghui@ccmu.edu.cn 'Department of Occupational Medicine and Toxicology, Beijing Institute of Respiratory Medicine, Beijing Chao-Yang Hospital, Capital Medical University, Worker's Stadium No.8, Chao-Yang District, Beijing 100020, China

${ }^{2}$ Institute of Infectious Diseases, Beijing Ditan Hospital, Capital Medical

University, Jingshundongjie 8, Beijing 100015, China

Full list of author information is available at the end of the article
}

Although it has been accepted that disturbed immune homeostasis plays a significant role in the pathogenesis and/or progression of IPF, the underlying mechanisms remains unclear. IPF has a different fibrotic process than connective tissue disease-related interstitial pneumonia (CTD-IP). It has been proposed that the devastating fibrotic response in IPF is driven by abnormally activated alveolar epithelial cells [2] rather than a chronic inflammatory process. Moreover, CTD-IP patients might benefit from corticosteroids and/or immunosuppressive agents, whereas IPF patients are unresponsive to immunosuppressants [1] and have few effective treatment options beyond lung transplantation [3].

Regulatory T cells (Tregs), a subpopulation of T cells, have immunosuppressive effects that maintain immune tolerance and immune response homeostasis [4]. 
Accumulated data have shown that Tregs contribute to maintaining immune homeostasis and are involved in a number of respiratory diseases. However, the effects of Tregs on lung fibrogenesis tend to conflict regarding their pro-fibrotic or anti-fibrotic role in pulmonary fibrosis [5-9]. On one hand, Tregs could interfere with upstream inflammatory events and indirectly decrease the development of fibrosis through inhibition of inflammatory and $T$ helper cell responses [7]. On the other hand, immunosuppressive Tregs are thought to possess profibrotic functions by secreting the most potent profibrotic cytokines, transforming growth factor (TGF)- $\beta_{1}$ and platelet-derived growth factor (PDGF)-B [5].

Early studies showed that $\mathrm{CD} 4^{+} / \mathrm{CD} 25^{\mathrm{hi}+} / \mathrm{Foxp}^{+}$ Tregs were retained in the lungs of bleomycin-treated $\mathrm{CCR}^{-1-}$ mice, which was consistent with an ameliorated remodelling response to bleomycin-induced injury in the lungs [8]. The number and function of Tregs was decreased in both peripheral blood (PB) and bronchoalveolar lavage (BAL) samples from patients with IPF compared to patients with CTD-IP and non-IPF lung diseases [10]. In contrast, other studies reported higher circulating Tregs in patients with rapidly progressive IPF [11, 12].

Most of the studies used $\mathrm{CD} 4^{+} \mathrm{CD} 25^{+} \mathrm{Foxp}^{+}$to define Tregs. However, recent research has demonstrated that these classical-defined Tregs are heterogeneous and separable into three functionally and phenotypically distinct subpopulations: $\mathrm{CD}_{4} 5 \mathrm{RA}^{+} / \mathrm{CD} 25^{++}$resting Tregs (rTregs) and CD45RA ${ }^{-} / \mathrm{CD}_{2} 5^{+++}$activated Tregs (aTregs), both of which are suppressive in vitro, and cytokine-secreting $\mathrm{CD}^{2} 5 \mathrm{RA}^{-} / \mathrm{CD}^{2} 5^{++} \mathrm{T}$ cells (Fr III), which are proinflammatory [13]. Based on this novel definition, recent studies have revealed the clinical relevance of subpopulations of Tregs in autoimmune diseases [14-16] and infectious diseases [17-19]. Recently, we reported that patients with chronic obstructive pulmonary disease (COPD) had decreased rTreg and aTreg cells but significantly increased Fr III cells in both PB and BAL compared with smokers [20], which indicated a disturbed homeostasis of Treg subpopulations in COPD. Here, by using the new identification strategy for the Treg subpopulations, we found that aTreg levels correlate with the disease severity of IPF, and we explored the disturbed adaptive immune response differences between COPD and CTD-IP.

\section{Methods}

\section{Study population}

We recruited 29 consecutive Chinese Han patients with newly diagnosed IPF, 19 patients with primary Sjögren's syndrome-related interstitial pneumonia (pSS-IP), and 23 healthy controls (HCs) at Beijing Chao-Yang Hospital, Capital Medical University, China (Table 1).

Diagnosis of IPF was based on the American Thoracic Society (ATS)/European Respiratory Society (ERS)/Japanese
Table 1 Demographics of the study participants

\begin{tabular}{lllll}
\hline & $\mathrm{HCS}$ & $\mathrm{pSS}-\mathrm{IP}$ & $\mathrm{IPF}$ & P-value $^{*}$ \\
\hline Subjects & $n=23$ & $n=19$ & $n=29$ & \\
Age, years & $66.1 \pm 8.6$ & $60.6 \pm 7.6$ & $65.1 \pm 6.2$ & 0.056 \\
Female/male, $\mathrm{n}$ & $12 / 11$ & $15 / 4$ & $1 / 28$ & 0.000 \\
Smoker/non-smoker, $\mathrm{n}$ & $10 / 13$ & $4 / 15$ & $27 / 2$ & 0.000 \\
$\mathrm{PaO}_{2}, \mathrm{mmHg}$ & $94.7 \pm 5.9$ & $85.1 \pm 16.6$ & $86.6 \pm 11.1$ & 0.061 \\
$\mathrm{FVC} \%$ predicted & $95.2 \pm 8.7$ & $71.0 \pm 13.1$ & $80.1 \pm 18.2$ & 0.000 \\
$\mathrm{FEV}_{1}, \%$ predicted & $89.3 \pm 10.0$ & $70.2 \pm 12.5$ & $84.1 \pm 18.0$ & 0.000 \\
$\mathrm{FEV}_{1} / \mathrm{FVC}$, \% & $85.2 \pm 3.9$ & $82.4 \pm 5.9$ & $81.6 \pm 5.8$ & 0.089 \\
$\mathrm{TLC}_{1} \%$ predicted & $88.8 \pm 5.0$ & $67.9 \pm 11.1$ & $75.6 \pm 11.6$ & 0.000 \\
$\mathrm{DLCO}, \%$ predicted & $88.3 \pm 4.9$ & $36.9 \pm 20.8$ & $43.3 \pm 16.5$ & 0.000
\end{tabular}

Data are presented as the means $\pm S D$ or $n .{ }^{*}: P$-value denotes statistical differences among the three groups; DLCO: diffusing capacity of the lung for carbon monoxide; $\mathrm{FEV}_{1}$ : forced expiratory volume in the first second; FVC: forced vital capacity; $\mathrm{PaO}_{2}$ : partial pressure of arterial oxygen; TLC: total lung capacity

Respiratory Society (JRS)/Latin American Thoracic Association (ALAT) statement [1]. Patients in the pSS-IP group were diagnosed with pSS by the current guideline [21]. Their chest HRCTs showed a nonspecific interstitial pneumonia pattern. Each patient was in a stable clinical and functional state. Patients who presented with signs of heart failure, acute pulmonary infection or pulmonary thromboembolism were excluded, as were those who were receiving treatment with corticosteroids and/or immunosuppressants at the time of our study. Healthy controls are volunteers recruited whose age matched with case groups, without evidence of age-related arterial hypertension, diabetes, cardiovascular disorders and cerebral vascular disease. The study protocol (No. 81370159) was approved by the Ethics Committee of Beijing Chao-Yang Hospital, and all participants provided written informed consent.

\section{Sample collection}

Peripheral blood (PB) samples were obtained from each subject and were processed to obtain peripheral blood mononuclear cells (PBMCs) by density centrifugation. BAL was performed and processed as previously described [22-24] in 7 patients with IPF and 12 patients with pSS-IP. Nine subjects underwent routine healthy examinations, received diagnostic bronchoscopy and showed normal BAL cytology [22]. BAL and PB samples were processed immediately after collection. BAL cell differentials of the study groups are shown in Table 2.

\section{Flow cytometry analysis}

Fresh PBMCs and BAL cells were stained with the following antibodies: CD45RA-FITC, CD45RA-APC, CD25-PE, CD25-PerCP-Cy5.5, CD4-FITC, CD4-PE-cy7, CD4-APC, $\mathrm{CD} 31-\mathrm{PE}$, and matched isotypic controls and incubated 
Table 2 Bronchoalveolar lavage cell differentials in the study population

\begin{tabular}{lllll}
\hline Characteristics & HCs & pSS-IP & IPF & $P$-value \\
\hline Subjects & $n=9$ & $n=12$ & $n=7$ & \\
Total cell counts, $\times 10^{6}$ & $6.4 \pm 4.7$ & $8.8 \pm 11.0$ & $3.6 \pm 3.4$ & 0.138 \\
Cell viability, \% & $86.5 \pm 6.5$ & $83.8 \pm 7.0$ & $84.8 \pm 8.3$ & 0.732 \\
Macrophage, \% & $95.0 \pm 12.2$ & $82.6 \pm 14.2$ & $91.0 \pm 19.1$ & 0.282 \\
Lymphocyte, \% & $3.3 \pm 2.4$ & $16.8 \pm 14.5$ & $6.2 \pm 3.8$ & 0.024 \\
Neutrophil, \% & $1.5 \pm 0.8$ & $1.5 \pm 2.1$ & $2.5 \pm 2.3$ & 0.557 \\
Eosinophil, \% & $0.4 \pm 0.3$ & $0.3 \pm 0.5$ & $0.3 \pm 0.8$ & 0.843
\end{tabular}

Values are presented as the means $\pm \mathrm{SD}$ or $\mathrm{n}$. ${ }^{*}: P$-value denotes statistical differences among the three groups; HCs healthy controls, $p S S-I P$ primary Sjögren's syndrome-related interstitial pneumonia, IPF idiopathic pulmonary fibrosis

for $30 \mathrm{~min}$ at $4{ }^{\circ} \mathrm{C}$ in dark room. For intracellular staining, cells were fixed, permeabilized, and stained with Foxp3-PE and Ki-67-FITC, according to the manufacturer's instructions. All antibodies were purchased from BD Biosciences or Pharmigen (San Jose, CA) and e-Bioscience (San Diego, CA). Data acquisition and analysis were performed with a FACSCalibur, which was equipped with CellQuest Pro software (BD Biosciences, San Jose, CA). Approximate $10^{5}$ cells were acquired for subsequent data analysis.

\section{Cell sorting and proliferation assay}

For functional assays, CD $25^{+} \mathrm{T}$ cells were firstly isolated by positive selection of PBMCs (obtained from $30 \mathrm{ml}$ whole blood) labeled with magnetic-bead conjugated antihuman CD25 mAbs using MACS MultiSort Kit according to manufacturer's instructions (Miltenyi) from IPF patients. Purified $\mathrm{CD} 25^{+} \mathrm{T}$ cells were stained with $\mathrm{CD} 4$, CD25, and CD45RA antibodies, and then sorted into CD4 ${ }^{+} \mathrm{CD} 25^{++} \mathrm{CD}_{4} 5 \mathrm{RA}^{+}$cells (rTregs, Fr I, $1 \times 10^{4}$ ), CD4 ${ }^{+} \mathrm{CD} 25^{+++} \mathrm{CD}^{+} 5 \mathrm{RA}^{-}$cells (aTregs, Fr II, $1 \times 10^{4}$ ), and CD4 ${ }^{+} \mathrm{CD} 25^{++} \mathrm{CD}^{+} 5 \mathrm{RA}^{-}$cells $\left(\mathrm{Fr}\right.$ III, $\left.1 \times 10^{4}\right)$ using a FACS Aria II flow cytometer (Becton Dickinson). The purity of the Treg subsets was more than $95 \%$. The $\mathrm{CD} 4^{+} \mathrm{CD} 25^{-}$ cells (responder $\mathrm{T}$ cells, $2 \times 10^{4}$ ) from healthy donors (10 ml whole blood) can also be isolated using magneticbeads conjugated anti-human CD25 mAbs and antihuman CD4 mAbs.

$\mathrm{CD} 4^{+} \mathrm{CD} 25^{-}$responder cells $\left(2 \times 10^{4}\right)$ from healthy donors were labeled with $1 \mu \mathrm{M}$ CFSE (Invitrogen, OR, USA) and were then cocultured with $\left(1 \times 10^{4}\right)$ unlabeled, sorted rTreg, aTreg and Fr III cells at a 1:2 Treg subpopulations $/ \mathrm{CD} 44^{+} \mathrm{CD} 25^{-}$responder cell ratio in anti-CD3 (5 $\mu \mathrm{g} / \mathrm{ml}$ OKT3 mAb; eBioscience) coated plates in the presence of soluble anti-CD28 $(5 \mu \mathrm{g} / \mathrm{ml}$; eBioscience) for $72 \mathrm{~h}$ at $37{ }^{\circ} \mathrm{C}$ and $5 \% \mathrm{CO}_{2}$ in complete medium (RPMI 1640 with 10\% fetal calf serum). CFSElabeled cells was assessed by flow cytometry.

\section{The gender-age-physiology (GAP) index}

The multidimensional GAP index is a simple and reliable tool for disease severity stratification in IPF. We calculated the GAP score for every IPF patient according to the method reported by Ley et al. [25].

\section{Statistical analysis}

All analyses were performed with SPSS for Windows V16.0 (Chicago, Illinois, USA). Values are presented as the mean \pm standard $(\mathrm{SD})$ or as the median and IQR when appropriate. Groups were compared using analysis of variance, Student's $t$-test, Wilcoxon rank-sum test, or a chi-square test as appropriate. Correlations were assessed using a Pearson correlation test or Spearman's rank test. A $p$ value $<0.05$ was considered significant.

\section{Results}

\section{Demographic characteristics}

The characteristics of 29 patients with IPF, 19 with pSSIP and 23 HCs are summarized in Table 1. Most (93\%) of the IPF patients had a history of smoking, but none were current smokers. Compared with the IPF group, more patients with pSS-IP were female $(p<0.001)$. Pulmonary function values did not significantly differ between the IPF and pSS-IP groups.

\section{Increased frequencies of circulating aTregs in patients with IPF}

We first investigated the percentages of circulating Tregs classically defined as $\mathrm{CD} 44^{+} \mathrm{CD} 25^{++}$in different groups. Compared with HCs, patients with IPF or pSS-IP did not have significant differences in the percentages of CD4 ${ }^{+} \mathrm{CD} 25^{++}$cells (HCs: $7.22 \pm 1.59 \%$; IPF: $7.70 \pm 2.68 \%$; pSSIP: $6.67 \pm 1.31 \%$; Fig. 1c).

Since it has been shown that the degree of Foxp3 expression is proportional to CD25 expression in circulating $\mathrm{CD} 4^{+} \mathrm{T}$ cells, we divided $\mathrm{CD} 4^{+} \mathrm{CD} 25^{++} \mathrm{T}$ cells into three subpopulations: rTregs $\left(\mathrm{CD} 45 \mathrm{RA}^{+} / \mathrm{CD} 25^{++}\right.$, Fr I), aTregs $\left(\mathrm{CD}^{2} 5 \mathrm{RA}^{-} / \mathrm{CD} 25^{+++}\right.$, Fr II) and the cytokinesecreting subpopulation $\left(\mathrm{CD}^{2} 5 \mathrm{RA}^{-} / \mathrm{CD} 25^{++}\right.$, Fr III), as described previously (Fig. 1a-d) [13, 17, 19]. We found that the patients with IPF or pSS-IP and HCs showed comparable percentages of Fr III cells (Fig. 1e). However, the patients with IPF and patients with pSS-IP exhibited significantly decreased percentages of rTreg fractions $(0.73 \pm 0.52 \%$ and $0.84 \pm 0.31 \%$, respectively $)$ when compared with HCs $(1.49 \pm 0.62 \%, p<0.001$; Fig. 1f). We also observed comparable percentages of circulating aTregs in patients with pSS-IP and healthy controls $(p=0.952)$. Strikingly, compared with patients with pSSIP and HCs, patients with IPF had an increase in the 
a

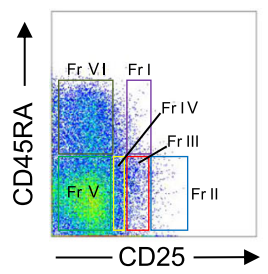

C

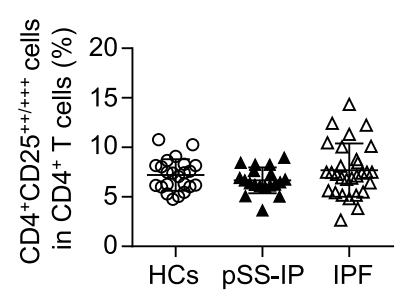

e

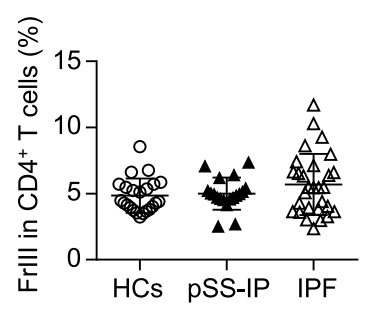

b

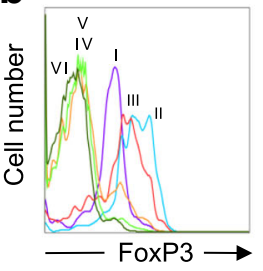

d

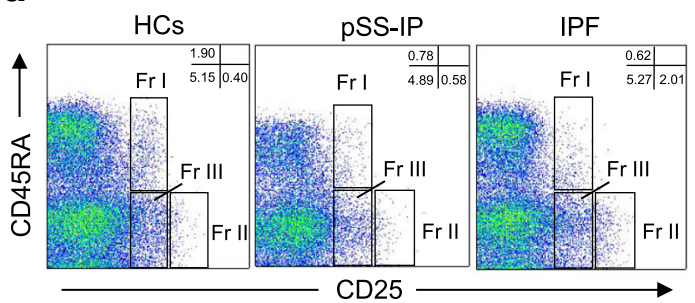

f

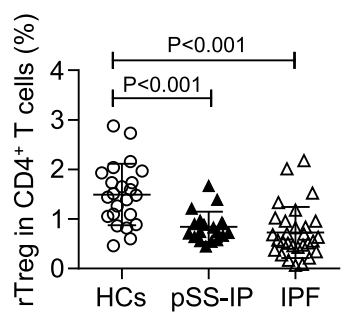

g

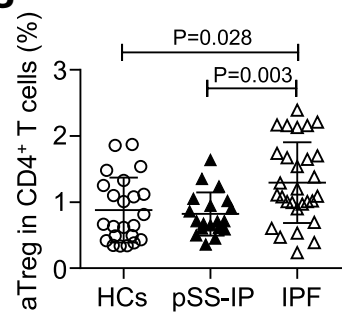

h

Responder alone Fr.I+ Responder Fr.II+ Responder Fr.III+ Responder

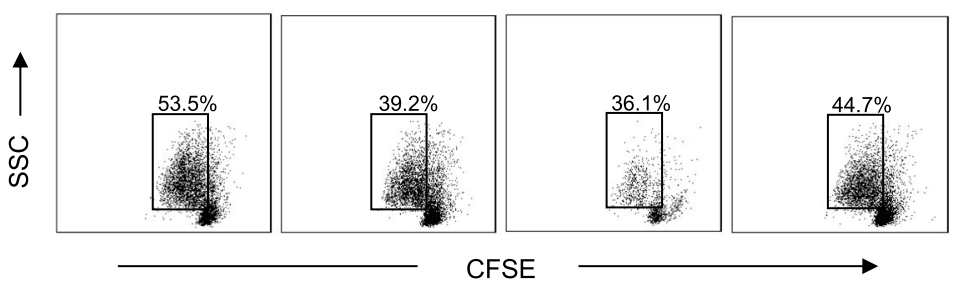

Fig. 1 The separation of $\mathrm{CD} 4^{+} / \mathrm{CD} 25^{+} / \mathrm{Foxp}^{+}$Treg cells into three subpopulations by their cell-surface molecules and in vitro suppressive activity. a Six subsets of $C D 4^{+} T$ cells defined by the expression of CD45RA and CD25. $\mathbf{b}$ The expression of Foxp3 in each fraction shown in (a). $\mathbf{c} C D 4^{+} \mathrm{CD} 25^{+}$ ${ }^{+/+++}$Tregs in $\mathrm{CD}^{+} \mathrm{T}$ cells in HCs and patients with pSS-IP or IPF. $\mathbf{d}$ Representative flow cytometric analysis of three subsets of $\mathrm{CD} 4^{+} \mathrm{CD} 25^{++} \mathrm{T}$ cells

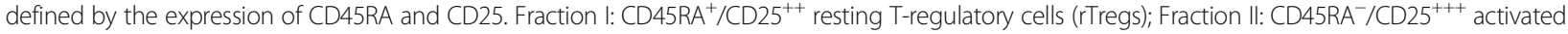
T-regulatory cells (aTregs); Fraction III: CD45RA $/$ CD25 $5^{++}$cytokine-secreting cells. e- $\mathbf{g}$ Percentages of circulating T-regulatory subpopulations (Fr. III, rTreg and aTreg cells) among CD4 ${ }^{+} \mathrm{T}$ cells in HCs and patients with pSS-IP or IPF. $\mathbf{h}$ CFSE-labelled CD4 $4^{+} \mathrm{CD} 25^{-} \mathrm{T}$ cells from HCs were cultured in the presence of soluble CD3 and CD28 either alone or with sorted Treg subpopulations from IPF patients at a 1:2 Treg subpopulations/ $\mathrm{CD} 4{ }^{+} \mathrm{CD} 25^{-}$responder cell ratio. The percentages of dividing cells are indicated. SSC: side scatter; CFSE: carboxyfluorescein diacetatesuccinimidyl ester; Responder cells represented $\mathrm{CD} 4^{+} / \mathrm{CD} 25^{-} \mathrm{T}$ cells

aTreg fraction $(1.29 \pm 0.61 \% ; p=0.003$ and $p=0.028$ respectively; Fig. 1g).

Next, in order to explore the immune functions of Treg subpopulations in patients with IPF, we performed an in vitro CFSE proliferation assay. Consistent with our previous study [20], we found that both rTregs and aTregs exhibited potent suppressive effects on responder cells, while Fr III cells showed a mild suppressive capacity compared with responders alone (Fig. 1h).
Impaired thymic output and enhanced proliferation account for imbalance of Treg subsets in IPF patients

To further investigate the imbalance of Treg subsets in patients with IPF, we assessed CD31 expression in Treg subpopulations, which is a marker for recent thymic emigrants [26] and enable the discrimination of recent thymic emigrant Treg cells from peripherally expanded naive Treg cells [27]. A decrease in the $\mathrm{CD} 31^{+}$fraction of rTreg cells was observed in IPF patients when 
compared to age-matched HCs $(27.79 \pm 13.81 \%$ vs $45.59 \pm 13.44 \%, p=0.005$; Fig. $2 \mathrm{a}$ ), suggesting that thymic production of Treg cells was affected in IPF patients and the impaired thymic output contributed to the decrease of rTreg cells. The maintenance of aTregs can be achieved by extensive turnover of the existing aTreg cells or by peripheral conversion of rTreg or nonTreg cells into aTreg cells. However, comparable percentages of the $\mathrm{CD} 31^{+}$fraction (mean $<10 \%$ ) were observed in aTregs from IPF patients and HCs (Fig. 2b), which might indicate only a small number of aTreg cells were converted from rTreg cells and contributed partly to the maintenance of aTreg cells pool. In the present study, we assessed the proliferative activity of Treg subsets by their expression of Ki-67, a nuclear protein that is expressed at a higher level in proliferating cells [13]. Compared with HCs, IPF patients displayed a slight increase in the percentages of $\mathrm{Ki}-67^{+}$cells in rTreg $(0.72 \pm 0.34 \%$ vs $3.98 \pm 2.44 \%, p=0.004$; Fig. 2 c) but a more dramatic increase in aTreg subsets $(4.54 \pm 2.86 \%$ vs $35.20 \pm 12.07 \%, p<0.001$; Fig. $2 \mathrm{~d}$ ), which indicates that aTregs in IPF patients are hyper-proliferative.

\section{Increased proportions of circulating aTregs correlated with disease severity in IPF patients}

We first investigated whether frequencies correlate with pulmonary function parameters, including forced vital

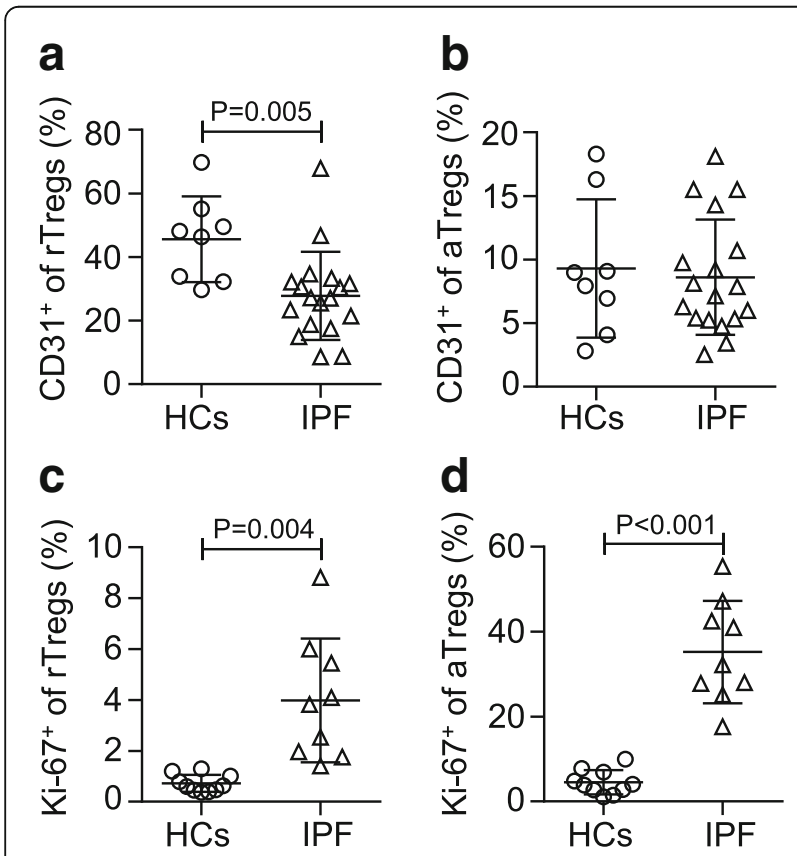

Fig. 2 Expression of CD31 and Ki-67 in Treg subpopulations. a, b The percentages of $\mathrm{CD}_{3} 1^{+}$cells among rTreg and aTreg in the peripheral blood of HCs and IPF patients. $\mathbf{c}$, $\mathbf{d}$ The percentages of Ki- $67^{+}$cells among rTreg and aTreg in the peripheral blood of HCs and IPF patients capacity (FVC) and DLCO that are used to predict IPF severity. As shown in Fig. 3, the percentages of aTregs in $\mathrm{CD} 4^{+} \mathrm{T}$ cells were negatively correlated with DLCO predicted values $\left(r={ }^{-} 0.475, p=0.016\right.$; Fig. 3a) but not with FVC $\%$ predicted values $\left(r={ }^{-} 0.257, p=0.225\right.$; Fig. 3b) in IPF patients. However, we did not notice correlation between the percentage of aTregs and predicted DLCO $\%$ in pSS-IP patients (data not shown). As reported by Ley et al. [25], GAP index and staging system has been used as a quick and simple screening method for informing prognosis in patients with IPF. We further noticed that the percentage of aTreg cells was positively correlated with GAP index in IPF patients $(r=0.488$, $p=0.018$; Fig. 3c).

\section{Increased proportions of aTregs in BAL from patients with IPF}

To further test whether disturbed homeostasis among Treg subpopulations also occurred in the lungs, we assessed the proportions of Treg subpopulations in BAL samples from 7 IPF patients, 12 pSS-IP patients and 9 HCs. In line with our previous study [20], the majority of Tregs in BAL were CD45RA ${ }^{-}$(Fig. 4a and c), which indicated that rTreg might only play limited roles in the local immunity of these patients.We used the combination of CD45RA and Foxp3 to separate Treg cells in BAL into two subsets, CD45RA Foxp $^{\text {low }}$ and CD45RA-Foxp $3^{\text {high }}$ (Fig. 4a and c). Differing from the results from circulating Fr III, both IPF patients and pSS-IP patients showed an increase in the percentages of Fr III fractions in BAL compared to HCs $(5.86 \pm 2.13 \% ; p=0.035$ and 0.030 , respectively) (Fig. 4d). More importantly, the results of aTregs from BAL were consistent with those from the PB. The patients with IPF showed significantly increased percentages of aTregs in BAL $(1.73 \pm 0.55 \%)$ than patients with pSS-IP $(0.57 \pm 0.40 \%, p=0.002)$ and HCs $(0.48 \pm 0.21 \%$, $p=0.002)$ (Fig. 4e).

\section{Discussion}

IPF is a chronic, progressive fibroproliferative interstitial pneumonia of unknown aetiology. Various studies have clarified the crucial roles of Tregs in many diseases, but contradictory results have been obtained from both murine models and IPF patients. Here, by using the new definition of Treg subpopulations, we showed that, compared with HCs and the patients with pSS-IP, patients with IPF had larger fractions of circulating aTregs, which was negatively correlated with DLCO predicted values and positively correlated with GAP index.

One of the most important findings of the present study is exploring a characterized disturbance of Treg subpopulations in patients with IPF. Consistent with the protective effects in pSS and other autoimmune diseases [28-31], a slight decrease in $\mathrm{CD} 4^{+} / \mathrm{CD} 25^{+}$Treg cells was 

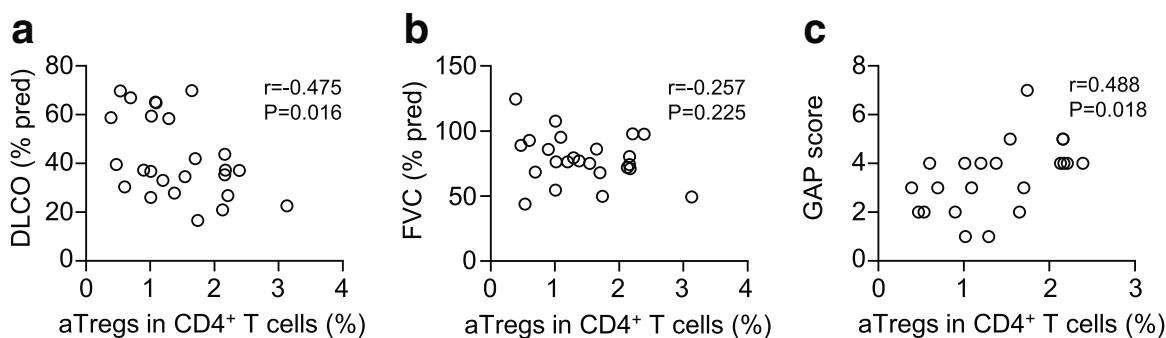

Fig. 3 Correlations of circulating aTreg fractions with lung function values and GAP index in patients with idiopathic pulmonary fibrosis. a aTreg cells vs DLCO \% predicted. $\mathbf{b}$ aTreg cells vs FVC \% predicted. c aTreg cells vs GAP score. DLCO: diffusing capacity of the lung for carbon monoxide; FVC: forced vital capacity; GAP: gender, age, and 2 lung physiology variables (FVC and DLCO)

a

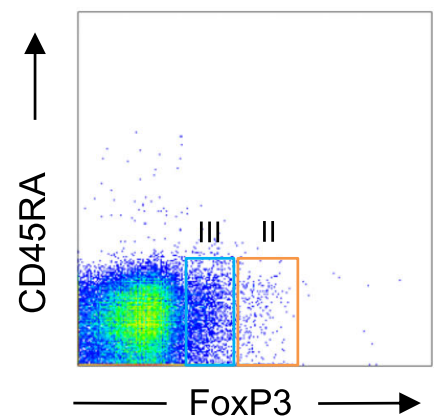

c

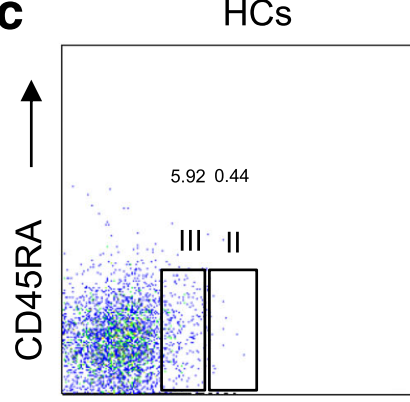

d

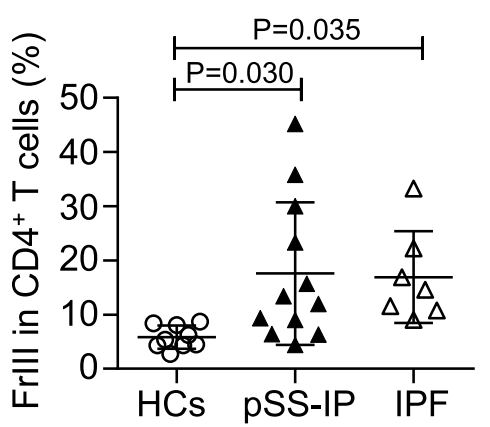

b

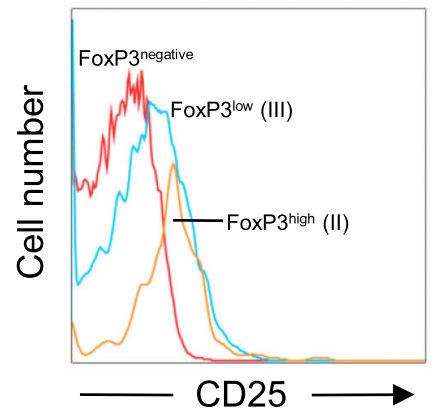

IPF

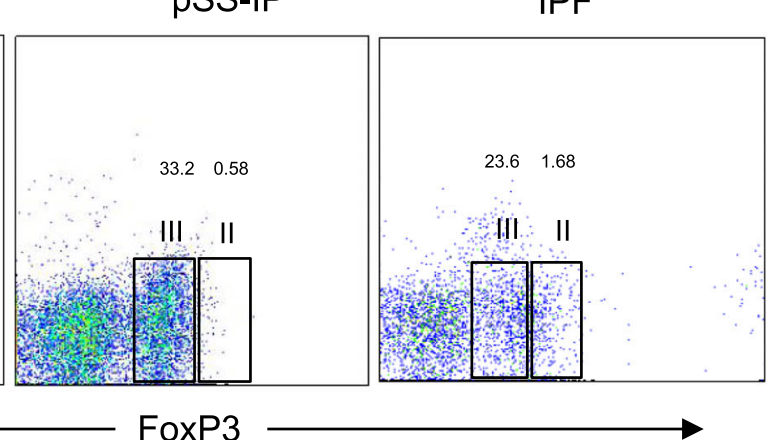

e

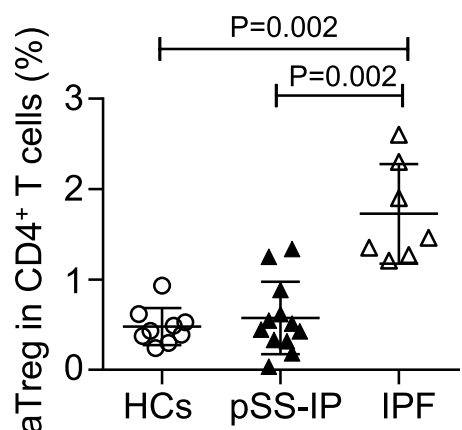

Fig. 4 Analysis of $\mathrm{CD}^{+} / \mathrm{CD} 25^{+} /$Foxp3 $^{+}$subpopulations from bronchoalveolar lavage (BAL) samples by cell-surface molecules and intracellular proteins. a Two subsets of Treg cells in BAL defined by the expression of CD45RA and Foxp3. Fraction II: CD45RA-Foxp3 ${ }^{\text {high }}$ activated T-regulatory cells (aTregs); Fraction III: CD45RA-Foxp3 ${ }^{\text {low }}$ cytokine-secreting cells. b The expression of CD25 in each fraction shown in (a). c Representative flow cytometric analysis of Treg subpopulations by the expression of CD45RA and Foxp3. d, e Percentages of Treg subpopulations among CD4 ${ }^{+} \mathrm{T}$ cells in the BAL of HCs and patients with PSS-IP or IPF 
observed in patients with pSS. Here, we also observed a significant decrease in the percentages of rTregs in patients with pSS-IP, while the percentages of aTregs remained comparable with the HCs. This finding indicated that inflammatory responses are related to a loss of rTregs in these patients. Similarly, IPF patients also had decreased percentages of rTregs. However, a dramatic increase in the percentages of aTregs was noticed. More importantly, a more dramatic increase in aTreg frequencies was observed in BAL samples of the patients with IPF. Thus, the disturbed homeostasis patterns between the IPF and pSS-IP patients were quite different. An increase in aTregs is a hallmark of disturbed immune homeostasis in IPF patients and might compensate for the loss of the rTreg fraction.

More importantly, although IPF patients display distinct defects in Treg subpopulations compared to patients with pSS-IP, a negative correlation was also seen between circulating aTregs and DLCO in IPF patients. Decreased rTregs and increased aTregs both showed detrimental effects in patients with pSS-IP and with IPF, respectively, which indicated that varying mechanisms contribute to fibrosis formation in these two diseases. In addition to a decreased number of Treg cells in pSS patients, in vitro functional assays showed decreased suppressive activity of $\mathrm{CD} 4^{+} / \mathrm{CD} 25^{+}$Treg cells [28]. Such defects in Treg cells might result in an overwhelming inflammatory response and subsequently cause tissue destruction in the lungs.

By contrast, in addition to an increase in aTreg cells, our in vitro functional analysis demonstrates that rTregs from IPF patients are functionally immunosuppressive. Our study showed that in addition to increased percentages of aTregs, inflammatory BAL Fr III cells were also increased. Despite the elevation of these two subpopulations, the aTreg/Fr III ratio in IPF patients was higher than in pSS-IP patients but comparable to that in HCs. From the above data, immune homeostasis seems to remain intact in IPF patients, although inflammatory and anti-inflammatory mechanisms were activated. This might explain why IPF patients are unresponsive to corticosteroids and/or immunosuppressive agents, whereas pSS-IP patients might benefit from them.

The disturbed homeostasis among Treg subpopulations in IPF may reflect cell derivation. All T cells, including these subpopulations, are derived from progenitors in the bone marrow and differentiate in the thymus [4]. Under various stimulations, rTregs can upregulate Foxp3 expression, differentiate to aTregs and continue to proliferate [13]. rTregs may represent the de novo generation of thymic lymphocytes, that the assessment of rTregs is possibly used to evaluate thymic Treg cell production. Howerver, rTregs can proliferate after thymic export while retaining their naive phenotype [32]. The surface expression of CD31 has been used as a direct marker of thymic output and enabled the discrimination of recent thymic emigrant Treg cells from peripherally expanded rTreg cells [27]. We found that the percentage of CD31 ${ }^{+}$ rTregs in IPF patients was significantly lower than in healthy controls, whereas $\mathrm{CD} 31^{+}$aTregs levels did not significantly differ between IPF patients and healthy controls. These results suggest an impaired thymic output of Tregs in IPF patients. The extensive turnover of the existing aTreg cells or by peripheral conversion of rTreg cells or non-Treg cells into aTreg cells have been proved to maintain the aTregs pool. The percentages of the $\mathrm{CD} 31^{+}$fraction in aTregs were low and in comparable level in IPF patients and HCs, indicating IPF might not affect conversion of rTregs to aTregs and contribute partly to the maintenance of aTreg cells pool. Actually, rTregs and aTregs represent essentially distinct populations from the genomic standpoint [33].In present study, we assessed the proliferative activity of individual Treg subsets by their expression of Ki-67 and found significantly increased percentage of $\mathrm{Ki}-67^{+}$fractions in aTregs and rTregs in IPF patients compared with healthy controls. Thus, hyperproliferation at least partly contributed to the high levels of circulating aTregs in IPF patients. These observations hint that a shift in the homeostatic composition of Treg subsets related to an impaired thymic-dependent de novo generation of recent thymic emigrant rTreg cells with a compensatory expansion of aTreg cells may contribute to imbalance of Treg subsets in IPF patients. What is noteworthy is that aTregs represent a highly differentiated population, characterized by short telomeres, inability to upregulate telomerase and susceptibility to apoptosis [34]. Therefore, aTregs have limited capacity for self-renewal, suggesting that it is unlikely that the aTregs pool is maintained through unintermittent turnover of existing aTregs. Some studies have suggested that aTregs can develop from Foxp3 ${ }^{-} / \mathrm{CD}^{+}{ }^{+}$non-Tregs [13] or from Fr III cells [19]. It is of interest to investigate whether there is an alternative pathway of generation of aTregs and how different pathways contribute to the maintenance of the aTregs pool in IPF patients.

Of note, it has been shown that Tregs can contribute to lung fibrosis by stimulating fibroblasts through the secretion of PDGF-B under non-inflammatory conditions and regulate detrimental T-cell activity during inflammation-related fibrosis $[5,6]$. Moreover, activated Tregs are capable of producing the potent pro-fibrotic cytokine TGF- $\beta_{1}$ [35]. Thus, it is not surprising that the percentage of circulating aTregs among $\mathrm{CD} 4^{+} \mathrm{T}$ cells in IPF patients was inversely correlated with DLCO predicted values. The multidimensional GAP (gender [G], age $[\mathrm{A}]$, and 2 lung physiology variables [P] [FVC and DLCO]) index and staging system is a simple method for informing prognosis, helping guide management 
decisions in patients with IPF [25]. Furthermore, the percentage of aTreg cells was positively correlated with GAP index in IPF patients.

Our study has some limitations that deserve comment. One limitation of this study is that the potential role of aTreg cells in chronic fibrotic lungs was not fully declared. Further research work is warranted to disclose whether the cells are per se that induce fibrogenesis or it is the pro-fibrotic microenvironment that shifts them towards a pro-fibrotic phenotype. Secondly, despite the appreciable effort of obtaining BAL samples, the results from the small size of the patients obtaining BAL may be compromised.

\section{Conclusions}

In conclusion, our study provided further evidence for the role of adaptive immunity in the pathogenesis of IPF and showed an imbalance among subpopulations of Tregs in IPF. Importantly, the increased aTregs in the PB correlate inversely with disease severity. Our study may suggest Tregs as potential future therapeutic targets by restoring their homeostasis. Treg subpopulations may be promising prognostic factors for IPF as well.

\begin{abstract}
Abbreviations
ALAT: Latin American Thoracic Association; aTregs: Activated Tregs; ATS: American Thoracic Society; BAL: Bronchoalveolar lavage; CFSE: Carboxyfluorescein diacetatesuccinimidyl ester; COPD: Chronic obstructive pulmonary disease; CTD-IP: Connective tissue disease-related interstitial pneumonia; DLCO: Diffusing capacity of the lung for carbon monoxide; ERS: European Respiratory Society; $F_{E V}$ : Forced expiratory volume in the first second; FVC: Forced vital capacity; HCs: Healthy controls; IPF: Idiopathic pulmonary fibrosis; JRS: Japanese Respiratory Society; $\mathrm{PaO}_{2}$ : Partial pressure of arterial oxygen; PB: Peripheral blood; PBMCs: Peripheral blood mononuclear cells; PBS: Phosphate-buffered saline; PDGF: Platelet-derived growth factor; pSS-IP: Primary Sjögren's syndromerelated interstitial pneumonia; rTregs: Resting Tregs; SSC: Side scatter; TGF: Transforming growth factor; TLC: Total lung capacity; Tregs: Regulatory T cells; UIP: usual Interstitial pneumonia
\end{abstract}

\section{Acknowledgements}

We thank all the patients who agreed to participate in the study and the staff of the Lung Function Lab and Bronchoscope Lab at Beijing Chao-Yang Hospital for their assistance.

\section{Funding}

This work was supported by the National Natural Science Foundation of China (No. 81370159) and the Beijing Natural Science Foundation of China (No. 7142058)

\section{Availability of data and materials}

Data and materials are available on reasonable request by contacting the first author Ziliang Hou (bright120@126.com).

\section{Authors' contributions}

ZLH performed all data collection, collected and processed samples, and wrote the manuscript. MHQ and YH performed laboratory-based assays. JYH was responsible for analysing the data. $\mathrm{HZ}$ contributed as a primary investigator and was responsible for designing the study and analysing the data. QY contributed as a primary investigator and was responsible for designing the study, recruiting the patients and writing the manuscript. All authors read and approved the final manuscript.

\section{Ethics approval and consent to participate}

Research ethics approval was obtained from Ethics Committee of Beijing Chao-Yang Hospital, Capital Medical University. All subjects provided written informed consent.

\section{Consent for publication}

Not applicable.

\section{Competing interests}

The authors declare that they have no conflict of interests.

\section{Publisher's Note}

Springer Nature remains neutral with regard to jurisdictional claims in published maps and institutional affiliations.

\section{Author details}

${ }^{1}$ Department of Occupational Medicine and Toxicology, Beijing Institute of Respiratory Medicine, Beijing Chao-Yang Hospital, Capital Medical University, Worker's Stadium No.8, Chao-Yang District, Beijing 100020, China. ${ }^{2}$ Institute of Infectious Diseases, Beijing Ditan Hospital, Capital Medical University, Jingshundongjie 8, Beijing 100015, China. ${ }^{3}$ Beijing Key Laboratory of Emerging and Reemerging Infectious Diseases, Beijing, China.

Received: 10 June 2017 Accepted: 31 August 2017

Published online: 08 September 2017

\section{References}

1. Raghu G, Collard HR, Egan JJ, Martinez FJ, Behr J, Brown KK, et al. An official ATS/ ERS/JRS/ALAT statement: idiopathic pulmonary fibrosis: evidence-based guidelines for diagnosis and management. Am J Respir Crit Care Med. 2011;183:788-824.

2. King TE, Pardo A, Selman M. Idiopathic pulmonary fibrosis. Lancet. 2011;378:1949-61.

3. Algar FJ, Espinosa D, Moreno P, Illana J, Cerezo F, Alvarez A, et al. Results of lung transplantation in idiopathic pulmonary fibrosis patients. Transplant Proc. 2010;42:3211-3.

4. Sakaguchi S. Naturally arising CD4+ regulatory t cells for immunologic self-tolerance and negative control of immune responses. Annu Rev Immunol. 2004;22:531-62.

5. Lo Re S, Lecocq M, Uwambayinema F, Yakoub Y, Delos M, Demoulin JB, et al. Platelet-derived growth factor-producing CD4+ Foxp3+ regulatory $T$ lymphocytes promote lung fibrosis. Am J Respir Crit Care Med. 2011;184:1270-81.

6. Liu F, Liu J, Weng D, Chen Y, Song L, He Q, et al. CD4+CD25+Foxp3+ regulatory $T$ cells depletion may attenuate the development of silicainduced lung fibrosis in mice. PLoS One. 2010;5:e15404.

7. Wilson MS, Wynn TA. Pulmonary fibrosis: pathogenesis, etiology and regulation. Mucosal Immunol. 2009;2:103-21.

8. Trujillo G, Hartigan AJ, Hogaboam CM. T regulatory cells and attenuated bleomycin-induced fibrosis in lungs of CCR7-/- mice. Fibrogenesis Tissue Repair. 2010;3:18.

9. Garibaldi BT, D'Alessio FR, Mock JR, Files DC, Chau E, Eto Y, et al. Regulatory $T$ cells reduce acute lung injury fibroproliferation by decreasing fibrocyte recruitment. Am J Respir Cell Mol Biol. 2013;48:35-43.

10. Kotsianidis I, Nakou E, Bouchliou I, Tzouvelekis A, Spanoudakis E, Steiropoulos $P$, et al. Global impairment of CD4+CD25+FOXP3+ regulatory T cells in idiopathic pulmonary fibrosis. Am J Respir Crit Care Med. 2009;179:1121-30.

11. Reilkoff RA, Peng H, Murray LA, Peng X, Russell T, Montgomery R, et al. Semaphorin $7 a+$ regulatory $T$ cells are associated with progressive idiopathic pulmonary fibrosis and are implicated in transforming growth factor-beta1induced pulmonary fibrosis. Am J Respir Crit Care Med. 2013;187:180-8.

12. Galati D, De Martino M, Trotta A, Rea G, Bruzzese D, Cicchitto G, et al. Peripheral depletion of NK cells and imbalance of the Treg/Th17 axis in idiopathic pulmonary fibrosis patients. Cytokine. 2014;66:119-26.

13. Miyara M, Yoshioka Y, Kitoh A, Shima T, Wing K, Niwa A, et al. Functional delineation and differentiation dynamics of human CD4+ T cells expressing the FoxP3 transcription factor. Immunity. 2009;30:899-911.

14. Liu X, Gao N, Li M, Xu D, Hou Y, Wang Q, et al. Elevated levels of CD4(+)CD25(+)FoxP3(+) T cells in systemic sclerosis patients contribute to the secretion of IL-17 and immunosuppression dysfunction. PLoS One. 2013;8:e64531. 
15. Pan X, Yuan X, Zheng Y, Wang W, Shan J, Lin F, et al. Increased CD45RA+ FoxP3(low) regulatory $T$ cells with impaired suppressive function in patients with systemic lupus erythematosus. PLoS One. 2012;7:e34662.

16. Kim JR, Chae JN, Kim SH, Ha JS. Subpopulations of regulatory T cells in rheumatoid arthritis, systemic lupus erythematosus, and Behcet's disease. J Korean Med Sci. 2012;27:1009-13.

17. Zhou H, Zhao H, Hao Y, Song C, Han J, Zhang J, et al. Excessive conversion and impaired thymic output contribute to disturbed regulatory T-cell homeostasis in AIDS patients with low CD4 cell counts. AIDS. 2013;27:1059-69.

18. Simonetta F, Lecuroux C, Girault I, Goujard C, Sinet M, Lambotte O, et al. Early and long-lasting alteration of effector CD45RA(-)Foxp3(high) regulatory T-cell homeostasis during HIV infection. J Infect Dis. 2012;205:1510-9.

19. Shi G, Han J, Liu G, Hao Y, Ma Y, Li T, et al. Expansion of activated regulatory T cells by myeloid-specific chemokines via an alternative pathway in CSF of bacterial meningitis patients. Eur J Immunol. 2014:44:420-30.

20. Hou J, Sun Y, Hao Y, Zhuo J, Liu X, Bai P, et al. Imbalance between subpopulations of regulatory T cells in COPD. Thorax. 2013;68:1131-9.

21. Vitali C, Bombardieri S, Jonsson R, Moutsopoulos HM, Alexander EL, Carsons $\mathrm{SE}$, et al. European study group on classification criteria for Sjögren's syndrome. Classification criteria for sjögren's syndrome: a revised version of the European criteria proposed by the American-European consensus group. Ann Rheum Dis. 2002;61:554-8.

22. Meyer KC, Raghu G, Baughman RP, Brown KK, Costabel U, du Bois RM, et al. An official American Thoracic Society clinical practice guideline: the clinical utility of bronchoalveolar lavage cellular analysis in interstitial lung disease. Am J Respir Crit Care Med. 2012;185:1004-14.

23. Report of the European Society of Pneumology Task Group. Technical recommendations and guidelines for bronchoalveolar lavage (BAL). Eur Respir J. 1989;2:561-85.

24. Tanino M, Betsuyaku T, Takeyabu K, Tanino Y, Yamaguchi E, Miyamoto K, et al. Increased levels of interleukin-8 in BAL fluid from smokers susceptible to pulmonary emphysema. Thorax. 2002;57:405-11.

25. Ley B, Ryerson CJ, Vittinghoff E, Ryu JH, Tomassetti S, Lee JS, et al. A multidimensional index and staging system for idiopathic pulmonary fibrosis. Ann Intern Med. 2012;156:684-91.

26. Kimmig S, Przybylski GK, Schmidt CA, Laurisch K, Mowes B, Radbruch A, et al. Two subsets of naive $T$ helper cells with distinct $T$ cell receptor excision circle content in human adult peripheral blood. J Exp Med. 2002;195:789-94.

27. Haas J, Fritzsching B, Trübswetter P, Korporal M, Milkova L, Fritz B, et al. Prevalence of newly generated naive regulatory $T$ cells (Treg) is critical for Treg suppressive function and determines Treg dysfunction in multiple sclerosis. J Immunol. 2007;179:1322-30.

28. Szodoray P, Papp G, Horvath IF, Barath S, Sipka S, Nakken B, et al. Cells with regulatory function of the innate and adaptive immune system in primary Sjogren's syndrome. Clin Exp Immunol. 2009;157:343-9.

29. Sarigul M, Yazisiz V, Bassorgun Cl, Ulker M, Avci AB, Erbasan F, et al. The numbers of Foxp3+ Treg cells are positively correlated with higher grade of infiltration at the salivary glands in primary Sjogren's syndrome. Lupus. 2010;19:138-45.

30. Alunno A, Petrillo MG, Nocentini G, Bistoni O, Bartoloni E, Caterbi S, et al. Characterization of a new regulatory CD4+ T cell subset in primary Sjogren's syndrome. Rheumatology (Oxford). 2013;52:1387-96.

31. Cooles FA, Isaacs JD, Anderson AE. Treg cells in rheumatoid arthritis: an update. Curr Rheumatol Rep. 2013;15:352.

32. Akbar AN, Vukmanovic-Stejic M, Taams LS, Macallan DC. The dynamic co-evolution of memory and regulatory CD4+ T cells in the periphery. Nat Rev Immunol. 2007;7:231-7.

33. Booth NJ, McQuaid AJ, Sobande T, Kissane S, Agius E, Jackson SE, et al. Different proliferative potential and migratory characteristics of human $\mathrm{CD}^{+}$regulatory ${ }^{\top}$ cells that express either CD45RA or CD45RO. J Immunol. 2010;184:4317-26.

34. Vukmanovic-Stejic M, Zhang Y, Cook JE, Fletcher JM, McQuaid A, Masters JE, et al. Human $\mathrm{CD}^{+} \mathrm{CD}_{2} 5^{\text {hi}} \mathrm{Foxp}^{+}$regulatory T cells are derived by rapid turnover of memory populations in vivo. J Clin Invest. 2006;116:2423-33.

35. Tran DQ, Andersson J, Wang R, Ramsey H, Unutmaz D, Shevach EM. GARP (LRRC32) is essential for the surface expression of latent TGF-beta on platelets and activated FOXP3+ regulatory T cells. Proc Natl Acad Sci U S A. 2009;106:13445-50.

\section{Submit your next manuscript to BioMed Central and we will help you at every step:}

- We accept pre-submission inquiries

- Our selector tool helps you to find the most relevant journal

- We provide round the clock customer support

- Convenient online submission

- Thorough peer review

- Inclusion in PubMed and all major indexing services

- Maximum visibility for your research

Submit your manuscript at www.biomedcentral.com/submit

Biomed Central 